\title{
LncRNA HOTAIR promotes the growth and metastasis of gastric cancer by sponging miR-1277-5p and upregulating COL5A1
}

\author{
Zhijian Wei $^{1} \cdot$ Lei Chen $^{2,3} \cdot$ Lei Meng $^{4} \oplus$ CWenxiu Han ${ }^{1} \cdot$ Lei Huang $^{1} \cdot$ Aman Xu $^{1}$
}

Received: 3 February 2020 / Accepted: 21 May 2020 / Published online: 24 June 2020

(c) The International Gastric Cancer Association and The Japanese Gastric Cancer Association 2020

\begin{abstract}
Background Emerging studies have shown that HOTAIR acts as an oncogene in gastric cancer (GC). However, its role in the extracellular matrix and in tumor immune infiltration remains unknown.

Methods HOTAIR and COL5A1 levels were analyzed by bioinformatics analysis and validated by qRT-PCR, western blotting and immunohistochemistry assays. The regulatory relationships between components of the HOTAIR/miR-1277-5p/ COL5A1 axis and the role of this axis in GC were predicted by bioinformatics analysis, and validated by in vitro and in vivo experiments. The correlation between COL5A1 and GC immune infiltration was assessed by bioinformatics analysis and a COL5A1-based predictive nomogram was established using the Stomach Adenocarcinoma dataset from The Cancer Genome Atlas.

Results We found that HOTAIR and COL5A1 were overexpressed in GC compared to normal controls, which predicted poor prognosis. The regulatory relationship of the HOTAIR/miR-1277-5p/COL5A1 axis in GC was demonstrated, and HOTAIR and COL5A1 were found to promote GC growth while miR-1277-5p exerted the reverse effects. In addition, COL5A1 was negatively associated with tumor purity but positively associated with immune infiltration, which suggested that COL5A1mediated GC growth may be partially mediated by the regulation of immune infiltration. Additionally, the established COL5A1-based nomogram showed that COL5A1 can serve as a prognostic biomarker in GC.

Conclusions HOTAIR regulates GC growth by sponging miR-1277-5p and upregulating COL5A1, and COL5A1-mediated GC cell proliferation may be mediated by effects on the tumor microenvironment, which provides novel targets for GC treatment.
\end{abstract}

Keywords Gastric cancer · HOTAIR · MiR-1277-5p · COL5A1 · Immune infiltration

\section{Introduction}

Zhijian Wei and Lei Chen contributed equally to the work.

Electronic supplementary material The online version of this article (https://doi.org/10.1007/s10120-020-01091-3) contains supplementary material, which is available to authorized users.

Wenxiu Han

hwxhbh@126.com

$\triangle$ Lei Huang

huangleizhenting@126.com

Aman Xu

xuaman166@sina.com

1 Department of General Surgery, The First Affiliated Hospital of Anhui Medical University, 218 JiXi Avenue, Hefei 230022, Anhui, PR China
Approximately, 1,033,700 newly diagnosed gastric cancer (GC) cases and 782,700 GC-related deaths occurred worldwide in 2018, which makes it the 5th most common malignancy and the 3rd most common cause of cancer-related death in both sexes combined [1]. Although early detection,

2 Department of Urology, The First Affiliated Hospital of Anhui Medical University, $218 \mathrm{JiXi}$ Avenue, Hefei 230022, Anhui, People's Republic of China

3 Institute of Urology, Anhui Medical University, $218 \mathrm{JiXi}$ Avenue, Hefei 230022, Anhui, People's Republic of China

4 Department of General Surgery, The Fourth Affiliated Hospital of Anhui Medical University, 100 HuaiHe Avenue, Hefei 230002, Anhui, People's Republic of China 
surgery, chemotherapy and molecular targeted therapy for GC have greatly progressed, the prognosis remains poor, with a 5-year survival rate of $30 \%$ for advanced GC and $75 \%$ for early GC due to inoperability and recurrence [2-5].

Long noncoding RNAs (lncRNAs), defined as those with lengths exceeding $200 \mathrm{bp}$, are involved in the initiation and development of tumors [6]. Homeobox transcript antisense intergenic RNA (HOTAIR), a trans-acting lncRNA with 2158 nucleotides and 6 exons, has been found to interact with polycomb repressive complex 2 , leading to epigenetic silencing and tumorigenesis of many cancer types, including breast cancer and bladder cancer [7]. In GC, accumulating evidence has revealed that HOTAIR exerts its biological function by interacting with microRNAs (miRNAs) to regulate gene expression and participate in molecular pathways of tumorigenesis $[8,9]$. Although the roles and underlying mechanisms of HOTAIR-mediated carcinogenesis in GC have been clarified, more novel functions of HOTAIR in $\mathrm{GC}$ need to be investigated.

The extracellular matrix (ECM), as a major component of the tumor environment, becomes disorganized in tumor formation and thus affects tumor development [10]. Collagen, a part of the ECM, is found to deposit greatly during tumorigenesis, and increased collagen deposition enhances tumor cell proliferation and immune cell infiltration [11-13]. Studies on collagen type $\mathrm{V} \alpha 1$ chain (COL5A1) indicate that COL5A1 may be a new prognostic factor for patients with lung adenocarcinoma and that the miR-29b/COL5A1 axis could regulate bone metabolism and fibrogenic reactions $[14,15]$. Bioinformatics analysis has revealed that COL5A1 is a prognostic indicator in GC prognosis [16], however, the role of COL5A1 in GC remains unknown. Hence, exploring the effects of COL5A1 on the occurrence and progression of $\mathrm{GC}$ and its underlying mechanisms is urgent.

In the present study, we found that HOTAIR and COL5A1 were overexpressed in GC cell lines and that overexpression of HOTAIR promoted the proliferation and metastasis of GC by sponging miR-1277-5p and upregulating COL5A1. In addition, overexpression of COL5A1-enhanced GC growth, possibly through facilitating immune infiltration, while miR-1277-5p mimics exerted the reverse effects on GC development. The established nomogram based on COL5A1 level indicated that COL5A1 was a prognostic factor for GC patients. Altogether, we concluded that the HOTAIR/miR-1277-5p axis regulates GC tumorigenesis by affecting COL5A1 levels, which may provide new targets for GC treatment.

\section{Methods}

\section{RNA sequencing and microarray data analysis}

GC gene expression data downloaded from Gene Expression Omnibus (GEO) and The Cancer Genome Atlas (TCGA) were analyzed to identify differentially expressed genes (DEGs) and lncRNAs (DElncRNAs). A heatmap of DElncRNAs was constructed with cut-off criteria of $\log _{2} \mathrm{FC}>2.0$ and adj. $P$ value $<0.05$, and volcano plots of DEGs were drawn with cut-off criteria of $\log _{2} \mid \mathrm{FCl}>1.0$ and adj. $P$ value $<0.05$. Starbase [17], GEPIA [18], Kaplan-Meier analysis and log-rank test [19] were applied to predict the expression and overall survival (OS) of HOTAIR and DEGs in GC patients. Additionally, the binding sites between miR1277-5p and its target genes were predicted by Starbase [17].

\section{Pathway enrichment and biological function analysis}

The protein-protein interaction (PPI) network of DEGs was obtained from the STRING database [20] and constructed by Cytoscape software (version 3.7.1). Molecular COmplex DEtection (MCODE) was used for cluster analysis, and Gene Ontology (GO) and Kyoto Encyclopedia of Genes and Genomes (KEGG) pathways were analyzed by ClueGO in Cytoscape software. Additionally, gene set enrichment analysis (GSEA) software (version 3.0) was used to identify pathways associated with COL5A1 in GC. The ESTIMATE algorithm was adopted to calculate the ESTIMATE, stromal and immune scores of GC tissues [21]. The Tumor IMmune Estimation Resource (TIMER) algorithm database was analyzed to explore the correlation between COL5A1 levels and 6 types of infiltrating immune cells [22].

\section{Cell culture}

All human GC and immortalized gastric cell lines purchased from the Type Culture Collection of the Chinese Academy of Sciences (Shanghai, China) were cultured in Dulbecco's modified Eagle medium (DMEM, Gibco, USA) added with $10 \%$ fetal bovine serum (FBS, HyClone, USA), $100 \mathrm{KU} / \mathrm{L}$ penicillin and $100 \mathrm{mg} / \mathrm{L}$ streptomycin. Cells were maintained in an incubator at $37{ }^{\circ} \mathrm{C}$ with $5 \% \mathrm{CO}_{2}$.

\section{Transfection}

GC cells were transfected with $0.4 \mu \mathrm{g}$ of small interfering RNAs (siRNAs, against HOTAIR and COL5A1) or miR1277-5p mimics or inhibitor (GenePharma, Shanghai, China) using Lipofectamine 2000 reagent (Invitrogen, USA). Overexpression of HOTAIR was achieved by transfecting 
$0.4 \mu \mathrm{g}$ of pcDNA3.1 vector by cloning HOTAIR or COL5A1 sequence into GC cells, which were cultured for $48 \mathrm{~h}$ before the following experiments. The sequences of si-HOTAIRs, si-COL5A1s, miR-1277-5p mimics and inhibitor and their negative control (NC) are listed in Table S1.

\section{Quantitative reverse transcription polymerase chain reaction (qRT-PCR)}

TRIzol reagent (Invitrogen, USA) was applied to extract all total RNAs, and the cDNAs of HOTAIR and miR1277-5p were synthesized by a reverse transcriptase kit (M-MLV) (Takara, Dalian, China). QRT-PCR was conducted on the Bio-Rad real-time PCR system (Bio-Rad, USA) using the SYBR Premix Ex TaqTM kit (Roche, Switzerland). The relative HOTAIR, COL5A1 and miR1277-5p were calculated by the $2^{-\Delta \Delta \mathrm{Ct}}$ formula, and GAPDH and U6 were regarded as the internal references. All primers are displayed in Table S2.

\section{Western blotting}

Equal amounts of protein samples $(30 \mu \mathrm{g})$ were extracted by RIPA lysis buffer (Sigma, USA) and loaded onto $5 \%$ and $10 \%$ sodium dodecyl sulfate polyacrylamide gels for protein separation. After transferring protein to the polyvinylidene fluoride membrane (Millipore, USA) and blocking the membrane with 5\% nonfat milk, the membrane was incubated with primary (1:1000, Abcam) and anti-rabbit secondary antibodies (1:5000, Thermo Fisher). The immune binding was detected using the ECL detection system (Peiqing Technology Co., Ltd, Shanghai, China). The optical density (OD) was normalized to that of $\beta$-actin (1:1000, Abcam) or GAPDH (1:2000, Abcam).

\section{Immunohistochemistry}

Human GC tissues were collected from patients who underwent gastrectomy in the First Affiliated Hospital of Anhui Medical University. The study was approved by the Ethics Committee of Anhui Medical University and written informed consent was obtained from these patients. After fixing with 4\% paraformaldehyde, embedding in paraffin and slicing specimens into $5-\mu \mathrm{m}$ thick sections, sections were deparaffinized and dehydrated. After antigen retrieval and endogenous peroxidase destruction, sections were incubated with anti-COL5A1 antibody overnight and then incubated with secondary antibody. Sections were counterstained with hematoxylin after treating horseradish peroxidase and 3,3'-diaminobenzidine. A light microscope was used to capture the images.

\section{MTT and CCK-8 assay}

A density of 10,000 cells per well were seeded in a 96-well plate to culture for $24 \mathrm{~h}$. After transfection for $0,24,48,72 \mathrm{~h}$, $10 \mu \mathrm{L}$ of MTT (3-(4, 5-dimethylthiazol-2-yl)-2, 5-diphenyltetrazolium-bromide, $5 \mathrm{mg} / \mathrm{mL}$, Ameresco, USA) was added to each well and cultured for $4 \mathrm{~h}$, and then the solution above was discarded and the crystal was dissolved with DMSO $(100 \mu \mathrm{L})$ for 15 min. Cell Counting Kit-8 (CCK8; Beyotime, Shanghai, China) was also used to detect cell proliferation. After transfection for $48 \mathrm{~h}, 10 \mu \mathrm{L}$ of CCK-8 reagent was added. The relative proliferation was calculated based on the absorbance of each well.

\section{Ethynyldeoxyuridine (EdU) proliferation assay}

After transfection, cell proliferation was assessed at 0, 24, 48 and $72 \mathrm{~h}$ using the BeyoClick ${ }^{\mathrm{TM}}$ EdU kit (Beyotime, Shanghai, China) according to the manufacturer's instructions. EdU-positive cells were observed under fluorescence microscopy.

\section{Transwell migration/invasion assay}

Transwell chambers coated with Matrigel (BD Biosciences, USA) were used for the invasion assay and chambers without Matrigel were used for the migration assay. Briefly, after seeding $2 \times 10^{5}$ transfected cells into chambers (Corning Incorporated, USA), the upper wells were supplemented with DMEM without serum and the lower wells contained DMEM with $10 \%$ FBS; the cells were incubated for $72 \mathrm{~h}$ at $37{ }^{\circ} \mathrm{C}$ with $5 \% \mathrm{CO}_{2}$. Then, the nonmigrating or noninvading cells were removed and the chambers were fixed with methanol and glacial acetic acid (3:1) for 30 min. Crystal violet was used to stain the cells for $15 \mathrm{~min}$. An inverted microscope (Olympus, Tokyo, Japan) was applied to capture and count cells.

\section{Wound healing assay}

Transfected cells $\left(5 \times 10^{5}\right.$ cells $)$ were seeded on the plates, and a $10-\mu \mathrm{L}$ sterile tip was used to scratch the wound when the cells covered the plates. Pictures were obtained under the microscope at 0 and $24 \mathrm{~h}$ to calculate the width of the wound.

\section{Dual-luciferase reporter assay}

PmirGLO-HOTAIR/COL5A1-wt/mut (Gene Create, Wuhan, China) was constructed by cloning wild type (wt) or mutant (mut) HOTAIR/COL5A1 into the pmirGLO vector (Promega, USA). PmirGLO-HOTAIR/COL5A1-wt/mut $(0.4 \mu \mathrm{g})$ was cotransfected with miR-1277-5p mimics or inhibitor 
$(0.5 \mu \mathrm{L}, 20 \mathrm{pmol} / \mathrm{L})$ into $293 \mathrm{~T}$ cells using Lipofectamine 2000 (Invitrogen, USA). The Dual Luciferase Reporter Assay System (Promega, USA) was utilized to detect the luciferase activity and then normalized to the Renilla luciferase activity.

\section{Xenograft tumor assay}

Female nude mice (4 weeks old, 300-350 g) purchased from the Animal Center of Anhui Medical University were randomly allocated to two groups: the si-HOTAIR group and the si-NC group ( $n=6$ per group). A total of $100 \mu \mathrm{L}$ of AGS cell suspension containing $2 \times 10^{6}$ cells transfected with si-HOTAIR or si-NC was subcutaneously injected into mice. Tumor size was measured from the fourteenth day after injection and then measured every 3 days. Measurement of mouse body weight was conducted every week. All mice were sacrificed at 1 month and tumors were completely detached from the mice for tumor weight measurement. H\&E staining and immunohistochemistry were performed for morphological observation and COL5A1 expression detection, respectively. HOTAIR, miR-1277-5p and COL5A1 levels were determined by qRT-PCR.

\section{Statistical analysis}

GraphPad Prism 8.0 (San Diego, CA), Statistical package for social science (SPSS, version 22.0, IBM Corp., Armonk, NY, USA) and $R$ software (version 3.6.1) were used to analyze all data that are expressed as the means \pm standard deviation (SD). Two-tailed Student's $t$ test was employed to evaluate the difference between two groups, and $P<0.05$ was considered significant.

The Stomach Adenocarcinoma (STAD) dataset from TCGA was processed by deleting blank values for further analysis, and the thresholds used to divide patients by age, tumor grade, TNM stage, T stage, $\mathrm{N}$ stage, $\mathrm{M}$ stage, and percentage of positive lymph nodes were in accordance with other previous studies [23-29]. Based on the results of the $\chi^{2}$ test and LASSO regression, some important variables were selected, and Cox regression analysis was used to construct a nomogram using the rms package in R software. The performance of the model was assessed using the concordance index (C-index) and was internally validated using the bootstrap method with 1000 iterations, and the calibration curve, decision curve and clinical impact curve were plotted to visualize the agreement and net benefit of our model.

\section{Results}

\section{HOTAIR is overexpressed in patients with GC and promotes the proliferation and metastasis of GC, which predicts a poor prognosis}

A total of 55 abnormally expressed lncRNAs were identified in the TCGA-STAD dataset (Fig. 1a), and HOTAIR levels were significantly increased in GC samples compared to control samples (Fig. 1b). The results of the Starbase and GEPIA database analysis were in consistent with the results above (Fig. S1a, b), which was confirmed by qRT-PCR assay that HOTAIR was overexpressed in GC cell lines (Fig. 1c). Survival analysis indicated that patients with high levels of HOTAIR had shorter survival times (Fig. 1d). Then, we performed MTT, Transwell and wound healing assays to further test the role of HOTAIR in GC. First, the pcDNAHOTAIR vector and three si-HOTAIRs were synthesized and transfected into AGS cells, and stably transfected cells were selected. As shown in Fig. 1e, the pcDNA-HOTAIR vector elevated HOTAIR levels, and three si-HOTAIRs knocked down HOTAIR expression effectively. MTT assay indicated that pcDNA-HOTAIR greatly promoted the proliferation of GC cells (Fig. 1f) and that si-HOTAIR-I/II exerted the reverse effects (Fig. 1g). The results of Transwell and wound healing assays revealed that cell migration, invasion and wound healing were increased in the pcDNA-HOTAIR group (Fig. 1h, i), in contrast, knockdown of HOTAIR presented the opposite effects (Fig. 1j, k).

\section{HOTAIR exerts its biological roles by sponging miR-1277-5p and upregulating COL5A1}

To elucidate the mechanisms underpinning HOTAIR-mediated cellular growth, we first plotted the volcano plots of four GSE datasets (GSE2685, GSE13911, GSE29272 and GSE54129) and a TCGA-STAD dataset, which illustrated DEGs in GC samples (Fig. 2a). After intersection analysis, 62 DEGs were identified (Fig. 2b), and a PPI network was constructed (Fig. 2c). Based on the MCODE cluster score, four clusters were established and we selected cluster $1($ score $=12.714)$ for further analysis (Fig. 2d). A total of 15 genes, including COL1A1, COL3A1, COL5A1, etc. were identified in cluster 1, and a correlation heatmap further revealed the relationship among these genes (Fig. S2a). GO and KEGG pathway analysis found that these genes were mainly enriched in extracellular matrix components, ECMreceptor interactions, etc. (Fig. 2e, f). Elevated expression of these 15 genes was negatively correlated with OS in GC patients (Fig. S3). Then, we identified COL5A1 as the downstream target gene of HOTAIR, and the qRT-PCR results indicated that the COL5A1 level was positively correlated 

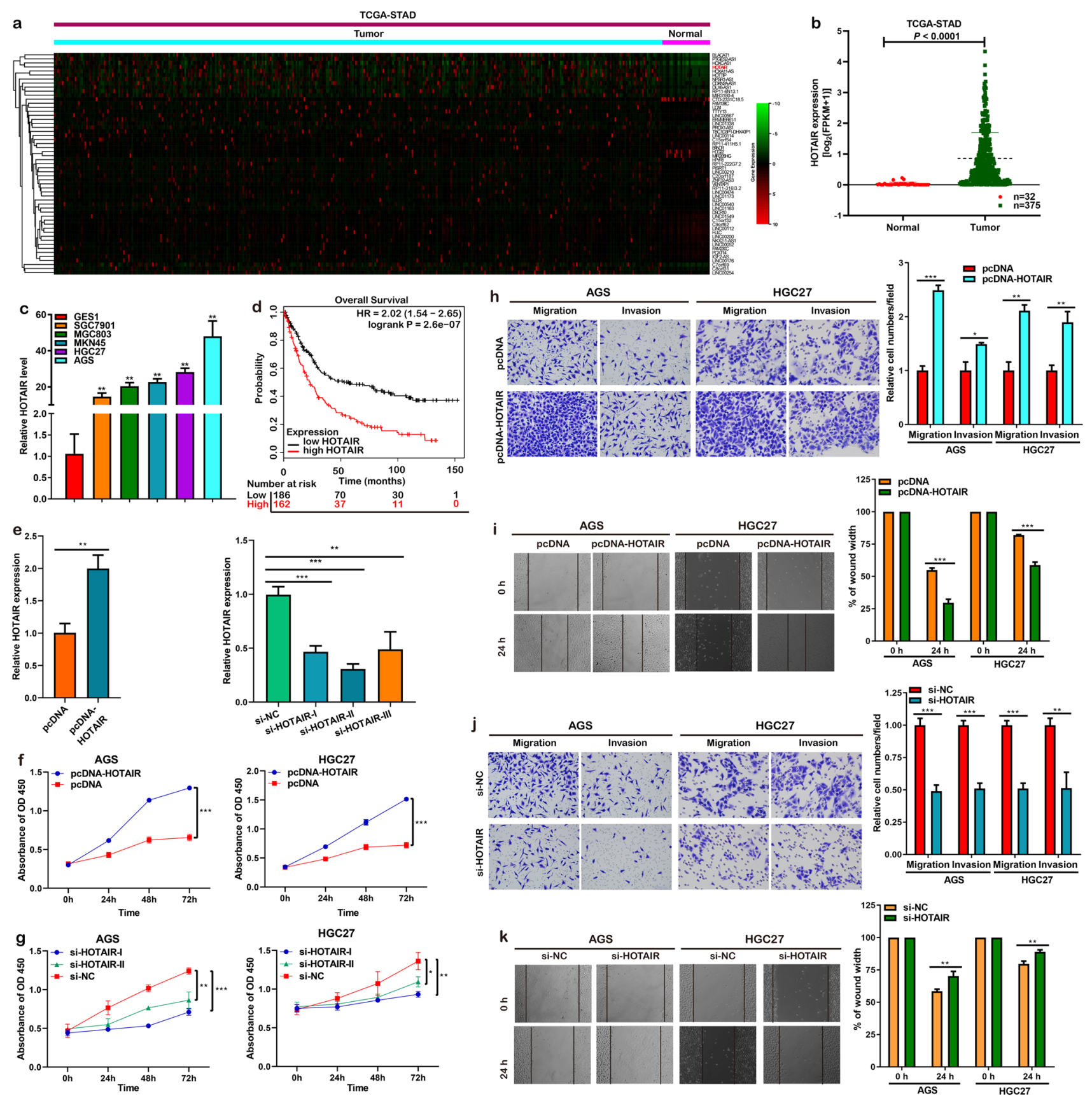

Fig. 1 HOTAIR was overexpressed in GC, which promoted the growth and metastasis of GC. a, b A Heatmap of differentially expressed lncRNAs in GC samples from the STAD dataset in TCGA, HOTAIR was found to be overexpressed in GC samples compared to normal samples. c HOTAIR levels were increased in GC cell lines by qRT-PCR. d Kaplan-Meier analysis showed that GC patients with higher HOTAIR expression had a poor prognosis. e QRT-PCR

with HOTAIR expression (Fig. 2g, $r=0.701, P=0.0012$ ). Previous studies elucidated that lncRNAs could function as ceRNAs by interacting with miRNAs to regulate tumorigenesis $[8,9,30]$. Hence, we speculated that HOTAIR may regulate COL5A1 by sponging miRNAs. The Venn assay demonstrated that pcDNA-HOTAIR and si-HOTAIRs effectively altered HOTAIR expression. $\mathbf{f}-\mathbf{k}$ Overexpression of HOTAIR promoted the proliferation, migration, invasion and wound healing of GC, while knockdown of HOTAIR exerted the reverse effects. STAD: stomach adenocarcinoma. Bars represent the mean \pm SD of three independent experiments. ${ }^{*} P<0.05,{ }^{* *} P<0.01,{ }^{* * * *} P<0.001$

diagram showed that HOTAIR and COL5A1 had 25 common miRNAs (Fig. S2b), which are listed in Table S3. As shown in Fig. S2c, binding sites between miR-1277-5p and HOTAIR or COL5A1 indicated that HOTAIR may regulate COL5A1 by sponging with miR-1277-5p. To confirm our 

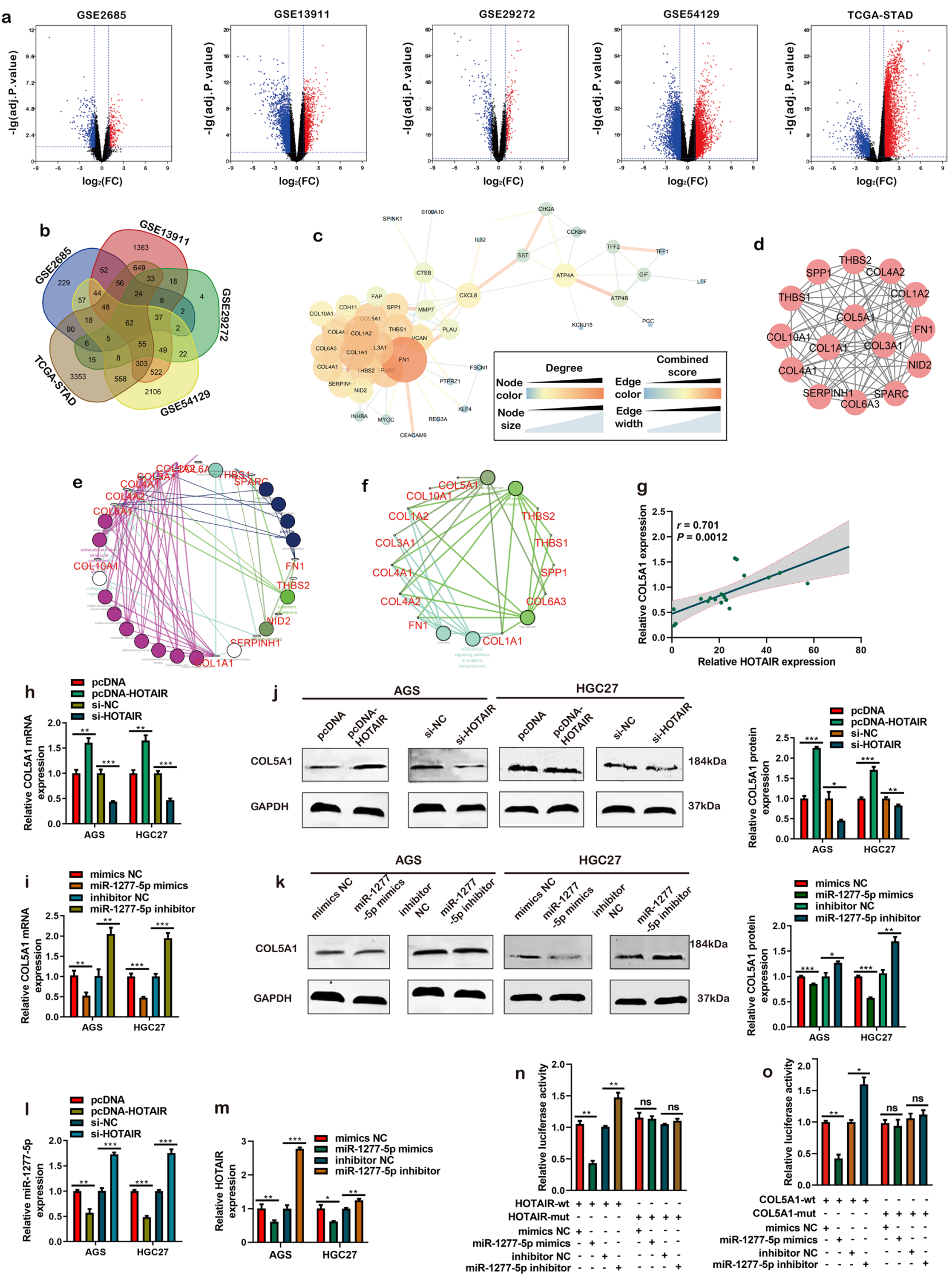
४Fig. 2 HOTAIR exerted its role in GC by sponging miR-1277-5p and upregulating COL5A1. a Volcano plots of four GSEs and STAD datasets showing DEGs in GC. b Sixty-two DEGs were identified in GC. c, d PPI of 62 DEGs by Cytoscape and 15 DEGs in cluster 1 by MCODE. e, f Results of GO and KEGG pathway analyses of 15 DEGs in cluster 1. g qRT-PCR assay indicated that the expression of COL5A1 was positively correlated with HOTAIR levels in GC cell lines. h-m The relationship among HOTAIR, miR-1277-5p and COL5A1 was demonstrated by qRT-PCR and western blotting assays. n, o Dual-luciferase reporter assay demonstrated that miR-1277-5p indeed interacted with HOTAIR and COL5A1. Bars represent the mean $\pm \mathrm{SD}$ of three independent experiments. ${ }^{*} P<0.05$; ${ }^{* *} P<0.01$; ${ }^{* * * *} P<0.001$

hypothesis, qRT-PCR was conducted. The COL5A1 level was elevated in the pcDNA-HOTAIR group but decreased in the si-HOTAIR group (Fig. 2h), and miR-1277-5p exerted reverse effects on COL5A1 expression (Fig. 2i). The western blotting assay results were in line with the qRT-PCR assay results (Fig. 2j, k). Additionally, HOTAIR and miR-1277-5p negatively regulated each other's expression (Fig. 2l, m).

Bioinformatics analysis predicted that direct binding sites existed between miR-1277-5p and HOTAIR or COL5A1, and a dual-luciferase reporter assay was performed to verify their interactions. HOTAIR/COL5A1-wt/mut sequences were synthesized, and pmirGLO-HOTAIR/COL5A1-wt/ mut was constructed (Fig. S2c). Compared with that in the control group, the luciferase activity decreased significantly in the HOTAIR/COL5A1-wt and miR-1277-5p mimics cotransfection group of $293 \mathrm{~T}$ cells, while greatly increased
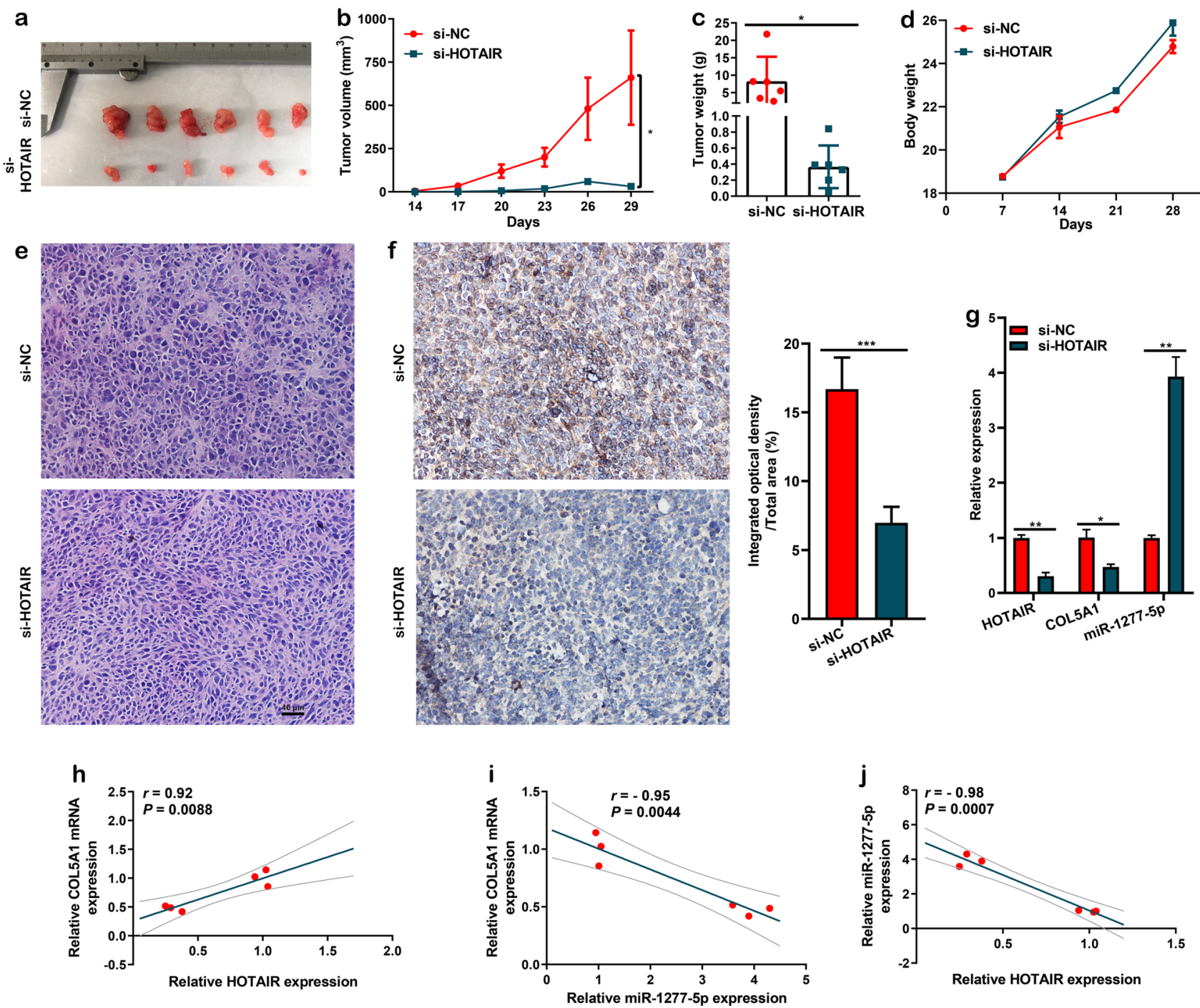

Fig. 3 Knockdown of HOTAIR suppressed GC growth in vivo. a Image of isolated tumors from nude mice. b-d Tumor volume and weight was attenuated when HOTAIR was knocked down, and the body weights of nude mice increased with increasing tumor volume. e Representative images of H\&E staining displaying the morphology of the tumor. $\mathbf{f}$ Representative images from immunohistochemistry assay indicated that COL5A1 was decreased in the presence of siHOTAIR. g-j Expression of HOTAIR, miR-1277-5p and COL5A1 in tumors was detected by qRT-PCR assay, and linear regression analysis was used to elucidate the relationship among them. Bars represent the mean \pm SD of six (b-d) or three (f, $\mathbf{g}$ ) independent experiments. ${ }^{*} P<0.05,{ }^{* *} P<0.01$ 


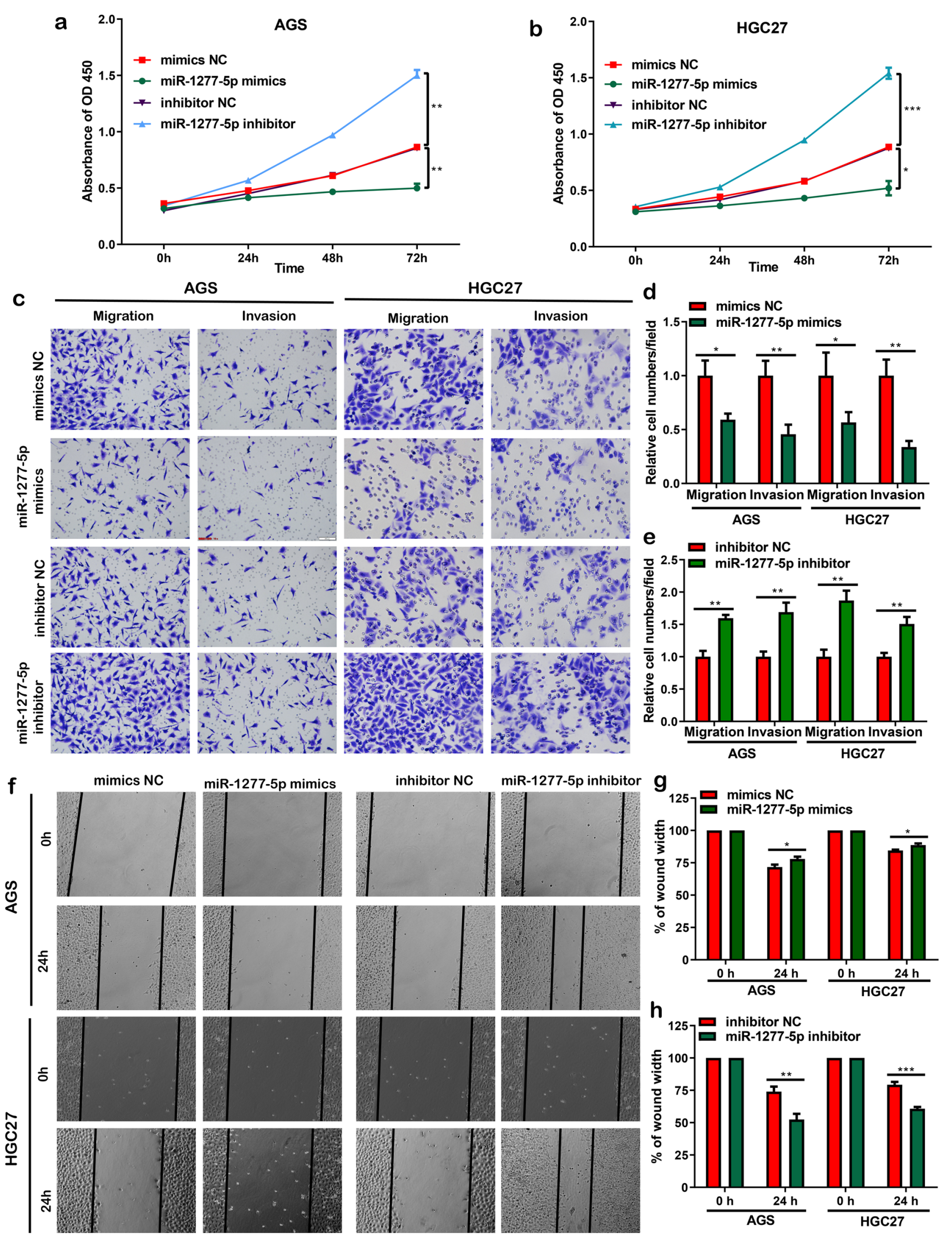


4Fig. 4 MiR-1277-5p attenuated the proliferation and metastasis of GC. a-h Overexpression of miR-1277-5p inhibited the proliferation, migration, invasion and wound healing of GC, while knockdown of miR-1277-5p exerted the reverse effects. Bars represent the mean \pm SD of three independent experiments. ${ }^{*} P<0.05,{ }^{* *} P<0.01$, ${ }^{* * * *} P<0.001$

luciferase activity was observed in the HOTAIR/COL5A1wt and miR-1277-5p inhibitor cotransfection group (Fig. 2n, o). Conversely, transfection of HOTAIR/COL5A1-mut abolished the effects above.

\section{Knockdown of HOTAIR restrains the growth of GC in vivo}

The oncogenic effect ofHOTAIR was confirmed by subcutaneously injecting mice with AGS cells transfected with siHOTAIR. As shown in Fig. 3a-d, tumor volume and weight increased more slowly in the si-HOTAIR group than in the control group. Images of H\&E staining showed the morphology of tumor tissues (Fig. 3e). The immunohistochemistry results showed that the level of COL5A1 was significantly decreased in the si-HOTAIR group (Fig. 3f). COL5A1 and HOTAIR expression was decreased in the presence of siHOTAIR, while miR-1277-5p levels were elevated when HOTAIR was knocked down (Fig. 3g). Moreover, linear regression analysis suggested that COL5A1 expression was positively correlated with the expression of HOTAIR (Fig. 3h, $r=0.92, P=0.0088$ ), while the expression of COL5A1 and HOTAIR was negatively correlated with the expression of miR-1277-5p (Fig. 3i, j, $r=-0.95, P=0.0044$; $r=-0.98, P=0.0007$ ).

\section{MiR-1277-5p inhibits the growth and metastasis of GC cells.}

To further explore the function of miR-1277-5p in GC development, in vitro experiments were conducted. Initially, the MTT assay revealed that miR-1277-5p mimics greatly suppressed the proliferation of AGS and HGC cells, while the miR-1277-5p inhibitor promoted proliferation (Fig. 4a, b). Additionally, the migration, invasion and wound healing abilities of GC cells were attenuated by upregulation of miR-1277-5p and enhanced by downregulation (Fig. 4c-h). Therefore, miR-1277-5p plays a vital role in the initiation and development of GC.

\section{High expression of COL5A1 is associated with the development and immune infiltration of GC}

The expression level of COL5A1 was increased in GC tissues according to analysis of the GEO and STAD datasets
(Fig. 5a-e), the COL5A1 level was higher in the high stromal and immune score group than in the low score group (Fig. 5f, g), and was positively correlated with the ESTIMATE score (Fig. 5h, $r=0.54, P<0.0001$ ) as well as the stromal and immune score (Fig. S4a, b). As shown in Fig. 5i, the GSEA results showed that cell cycle, DNA replication and pathways in cancer, etc., were enriched in tissues with high COL5A1, which was validated by analysis of the TCGA-STAD dataset (Fig. S4c). In addition, we found that pathways of the immune-related pathway were also enriched (Fig. S4d), and analysis of the TIMER algorithm validated that COL5A1 expression was negatively correlated with tumor purity but positively correlated with $\mathrm{CD} 8+\mathrm{T}$ cell, CD4 + T cell, macrophage, neutrophil and dendritic cell infiltration (Fig. S4e). To verify the findings above, we first found that COL5A1 was significantly elevated in GC tissues and cells (Fig. 5j-1). After confirming that siCOL5A1s effectively downregulated COL5A1 by western blotting and qRT-PCR assay (Fig. 5m, n), the CCK-8 assay was conducted, and the results showed that knockdown of COL5A1 greatly repressed the growth of AGS and HGC27 cells (Fig. 5o).

\section{MiR-1277-5p rescues the oncogenic effects of HOTAIR on GC by regulating COL5A1}

We cotransfected si-HOTAIR and miR-1277-5p inhibitor into GC cells and found that the miR-1277-5p inhibitor rescued the decrease in cell proliferation mediated by siHOTAIR (Fig. 6a), and the results of the wound healing assay were consistent with those of the EdU assay (Fig. 6b, c). Simultaneously, the miR-1277-5p inhibitor was able to restore the effects of si-HOTAIR on HOTAIR and COL5A1 expression (Fig. 6d, e). In addition, miR-1277-5p mimics and pcDNA-COL5A1 were cotransfected into AGS and HGC27 cells, and the results showed that pcDNA-COL5A1 rescued the effects of miR-1277-5p mimics on COL5A1 expression (Fig. 6f). Taken together, we could conclude that HOTAIR upregulated COL5A1 by sponging miR-1277-5p, which disrupted the normal ECM to change the GC microenvironment, promoting immune infiltration, proliferation and metastasis of GC (Fig. 6g).

\section{Prognostic nomogram for OS}

To further explore the role of COL5A1 in OS, a COL5A1based nomogram was formulated to predict the 1/3/5-year survival probability. As displayed in Table S4, tumor grade and $\mathrm{T}$ stage were significantly associated with COL5A1 expression. In addition, 8 variables [COL5A1, HOTAIR, gender, T stage, TNM stage, age, positive lymph nodes (positive LNs (\%)] and residual tumor) were screened out by LASSO regression (Fig. 7a, b). Then, we used age, gender, 

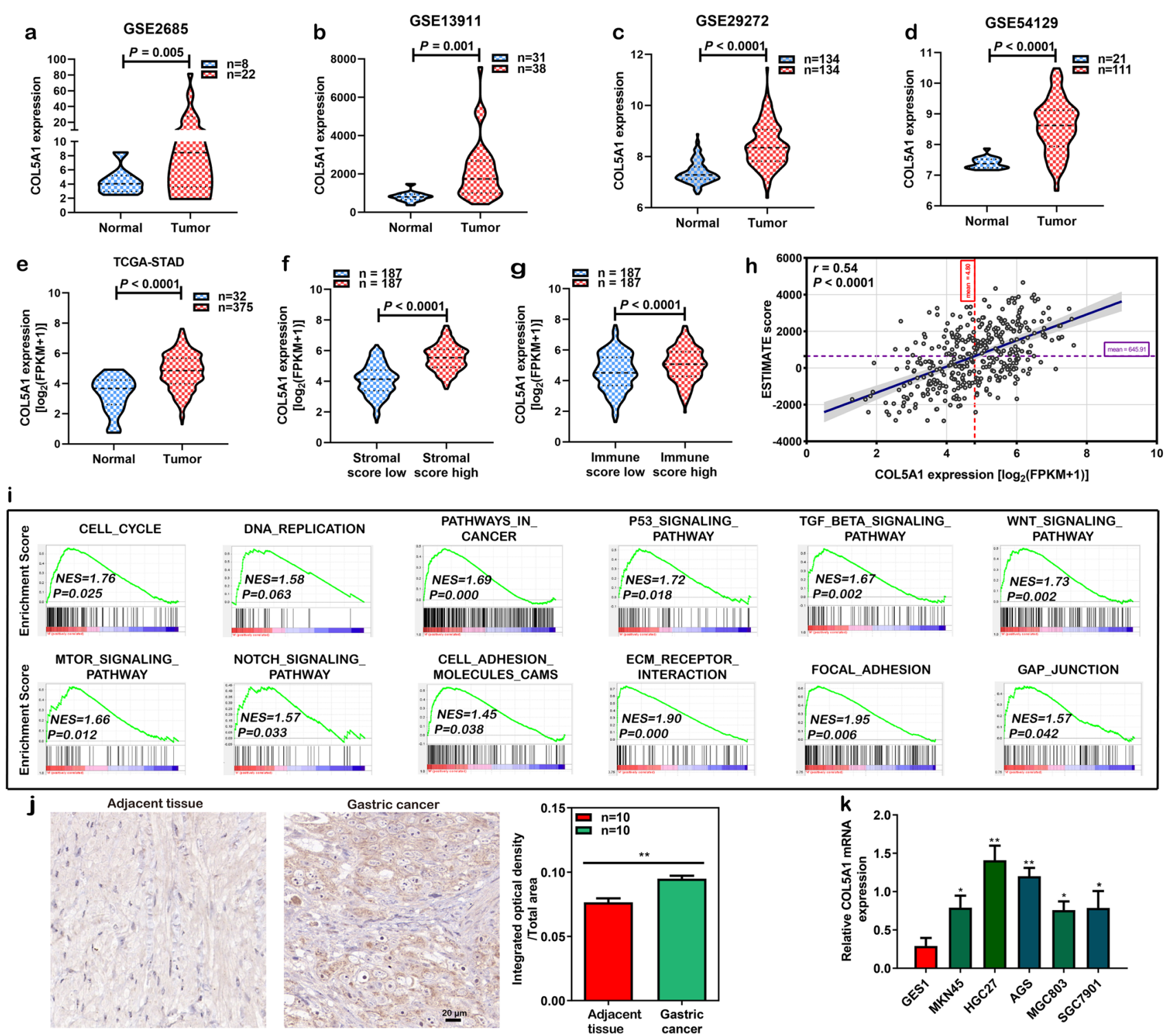

core low score high

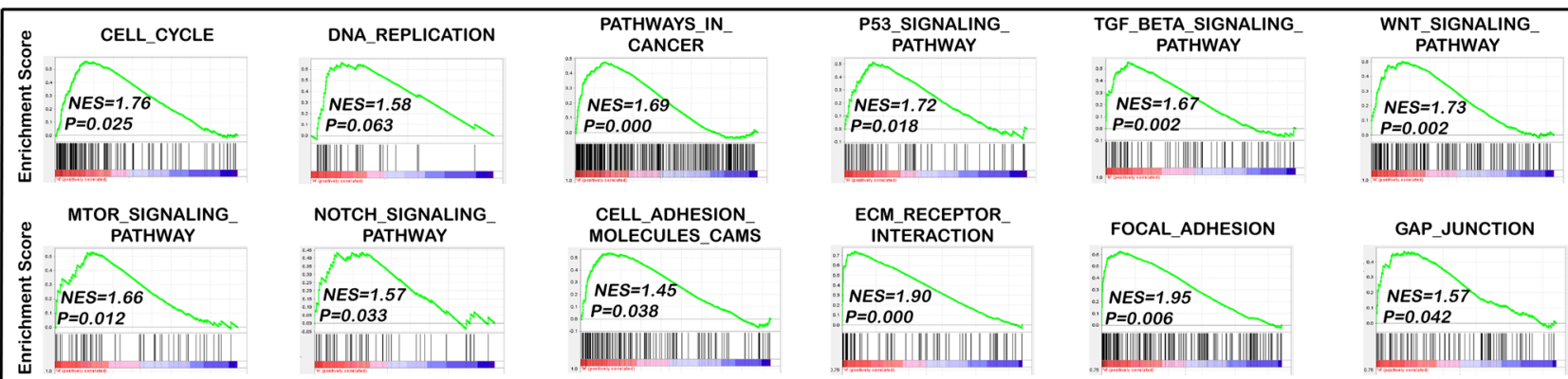

1
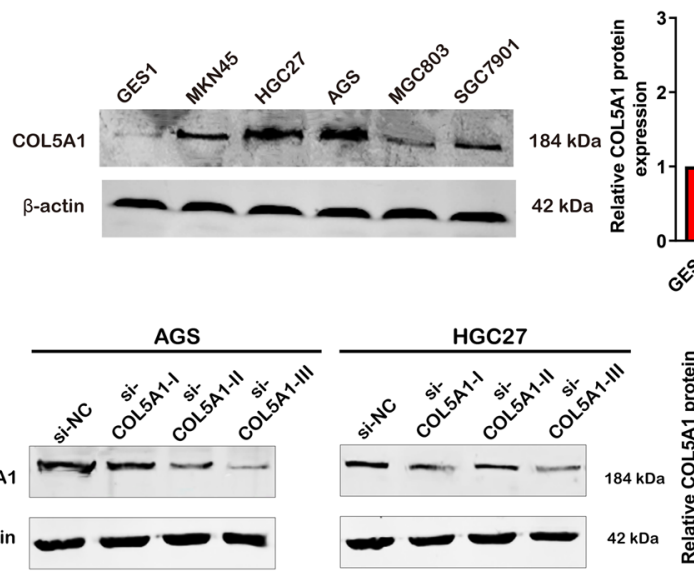
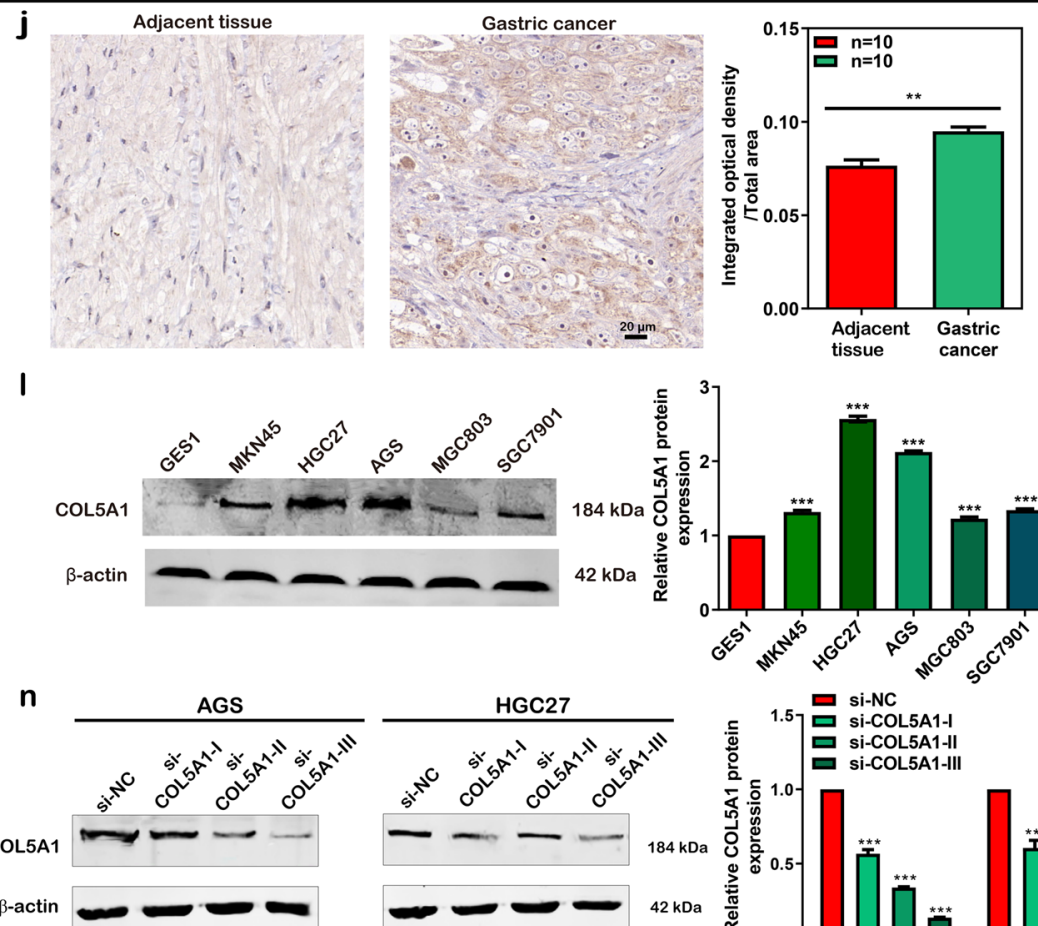
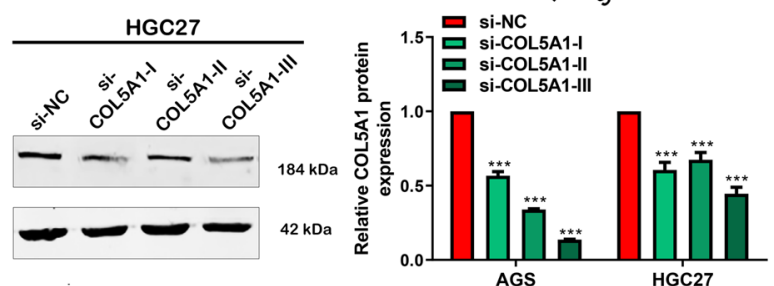
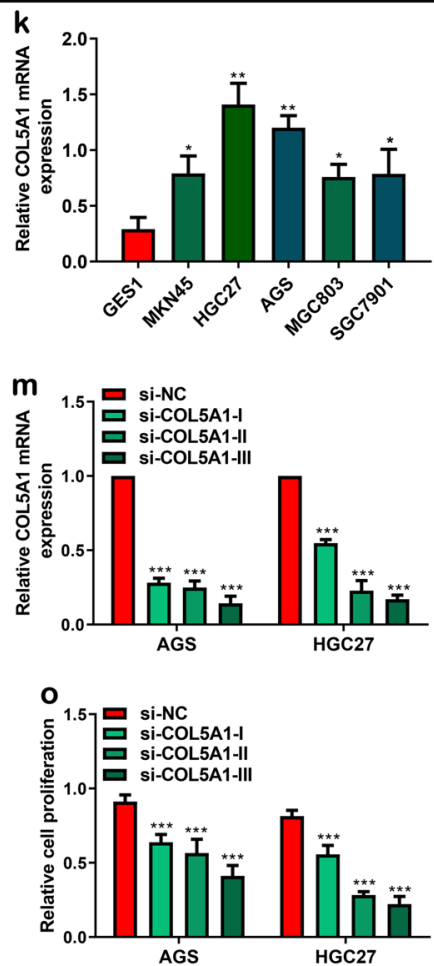
4Fig. 5 COL5A1 was related to the progression and immune infiltration of GC. a-e Bioinformatics analysis suggested that COL5A1 was significantly increased in GC samples. $\mathbf{f}-\mathbf{h}$ COL5A1 was found to be greatly elevated in the high stromal and immune score group, and the COL5A1 level was positively correlated with the ESTIMATE score. i GSEA was performed to visualize the correlation between COL5A1 levels and gene sets related to the cell cycle, DNA replication, etc. j-l Results of immunohistochemistry, qRT-PCR and western blotting assays indicated that COL5A1 was greatly increased in GC samples and cell lines. m-o COL5A1 levels were effectively downregulated by si-COL5A1s and knockdown of COL5A1 significantly attenuated GC growth. Bars represent the mean $\pm \mathrm{SD}$ of three independent experiments. ${ }^{*} P<0.05,{ }^{* *} P<0.01,{ }^{* * *} P<0.001$

tumor grade, positive LNs (\%) and COL5A1 to build the prognostic nomogram (Fig. 7c). The C-index for OS prediction was 0.69 (95\% CI 0.63-0.74), which was confirmed to be 0.67 by the bootstrap method. The calibration curves visualized the good agreement between the predicted OS and actually observed OS (Fig. 7d-f). Moreover, decision curve and clinical impact curve graphically suggested the clinical usefulness of the model based on a continuous threshold probability for OS prediction that the established model provided good net benefit (Fig. 7g, h).

\section{Discussion}

In our study, we explored the role of the HOTAIR/miR1277-5p/COL5A1 axis in GC and its underlying mechanisms. Our major findings were as follows: (i) HOTAIR was highly expressed in GC cells and enhanced the proliferation and metastasis of GC, which was mediated by sponging miR-1277-5p and upregulating COL5A1; (ii) miR-1277-5p suppressed the growth and metastasis of GC, while COL5A1 promoted the growth of GC, and COL5A1 was positively correlated with GC immune infiltration; (iii) miR-1277-5p reversed the oncogenic effects of HOTAIR on GC by regulating COL5A1; and (iv) the COL5A1-based nomogram demonstrated that COL5A1 was a prognostic factor in patients with GC. Taken together, these findings elucidated that HOTAIR upregulated COL5A1 expression by sponging miR-1277-5p to promote the progression of GC, and intervening collagen (COL5A1) expression by lncRNAs may provide new insights into the treatment of GC.

As one of the most common malignancies and the 3rd leading cause of cancer-related death, GC imposes a heavy burden on patients and society [1]. Hence, exploring reliable biomarkers and therapeutic targets to improve the early diagnosis and prognosis for patients with GC is essential. Increasing evidence indicates that HOTAIR is engaged in various cancer processes, including epigenetic activation or silencing and ceRNA networks [31]. For example, the HOTAIR/miR-331-3p/HER2 and HOTAIR/miR-217 axes can enhance the growth, invasion and chemotherapy resistance of GC $[8,9]$. In this study, we further demonstrated that HOTAIR promoted tumorigenesis and development of GC, which represented a poor prognosis in patients with GC. Interestingly, we found a novel miRNA (miR-1277-5p) that was engaged in HOTAIR-mediated GC progression, and HOTAIR regulated GC development by sponging miR-1277-5p.

Previous studies reported that the ECM is involved in tumorigenesis via processes including cell migration and proliferation [12, 32] and EMT [33]. Notably, collagen-containing fibers facilitated the rapid migration of tumor cells, and increased collagen density enhanced the proliferation and migration of tumor cells [12]. COL1 and COL11A1 have been proven to promote the malignant phenotype and progression of pancreatic ductal adenocarcinoma and head and neck squamous cell cancer [34-36]. Therefore, collagen exerts a regulatory role in the growth and invasion of tumors. Accumulating research indicates that COL5A1 may be a new prognostic factor for patients with lung adenocarcinoma [14]. In addition, a high level of COL5A1 accelerates the growth and progression of renal cell carcinoma and breast cancer [37, 38], which reminds us that ECM may play an important role in GC. In our study, we established an interaction network of ECM structural constituents, which were negatively related to the OS of GC patients. Additionally, bioinformatics analysis indicated that GC samples with high COL5A1 expression were positively associated with the cell cycle, DNA replication, etc. We further demonstrated that COL5A1 was highly expressed in GC cells and tissues, and knockdown of COL5A1 repressed the proliferation of GC cells, which was regulated by the HOTAIR/miR-1277-5p axis. HOTAIR-mediated cellular proliferation and metastasis was achieved by sponging miR-1277-5p and upregulating COL5A1.

Recently, increasing evidence has shown that tumor immune infiltration is a promising target of tumor treatment $[39,40]$, and collagen promotes the infiltration of immune cells in the tumor microenvironment to inhibit macrophages from killing cancer cells [10]. Coincidentally, our results suggested that COL5A1 was positively related to stromal, immune and ESTIMATE scores, implying that COL5A1 was negatively associated with tumor purity, an index representing the proportion of tumor cells in the tumor microenvironment. Specifically, we found that COL5A1 expression was negatively correlated with GC purity but positively correlated with CD8 + T cell, CD4 + T cell, macrophage, neutrophil and dendritic cell infiltration. Moreover, GC samples with high COL5A1 levels were positively associated with immune-related pathways including leukocyte transendothelial migration and natural killer cell-mediated cytotoxicity. Combined with previous studies showing that low tumor purity predicted poor prognosis in colon cancer, kidney renal clear cell carcinoma and lower grade glioma [41, 42], 


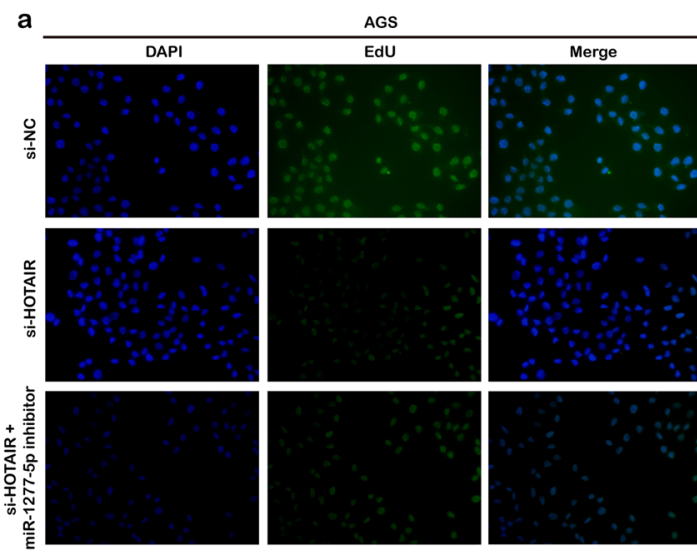

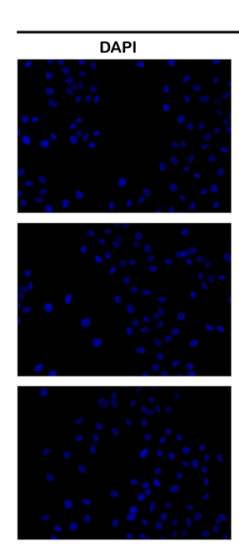

C
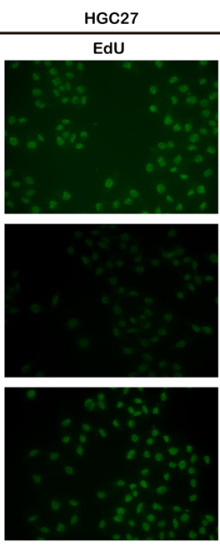

HGC27
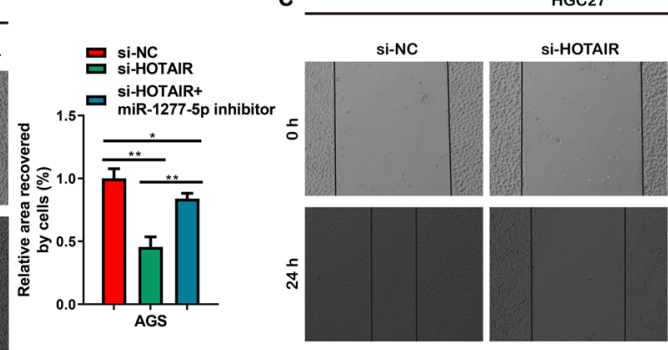
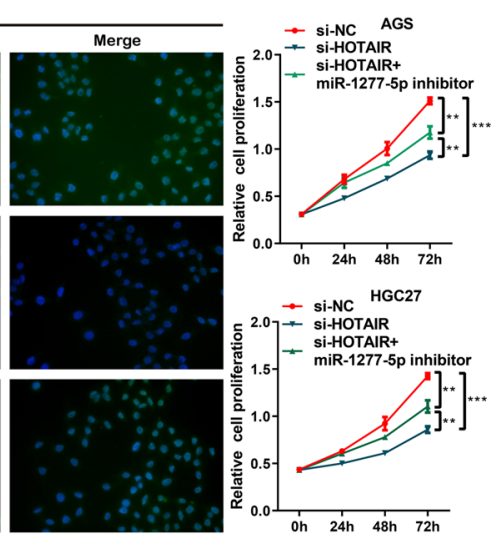

b

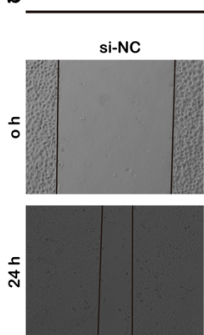

Si-HOTAIR
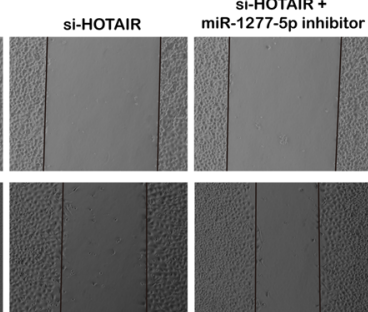

d
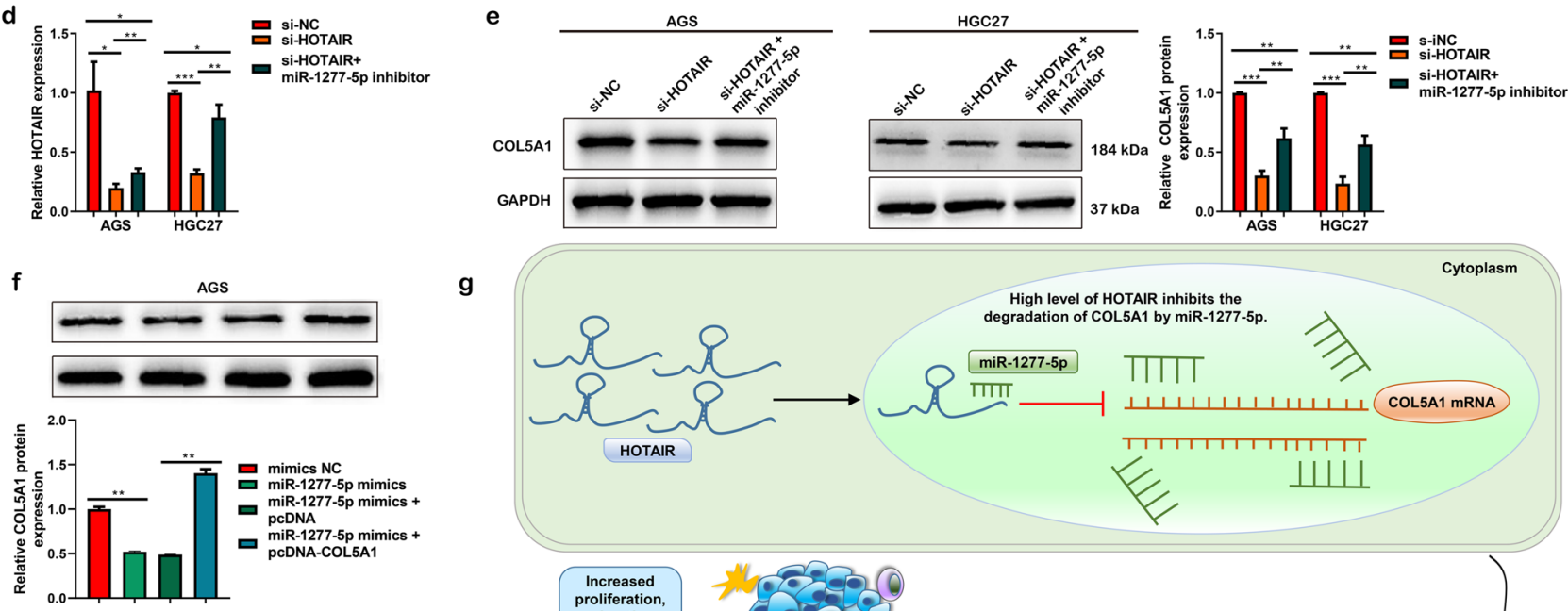

g
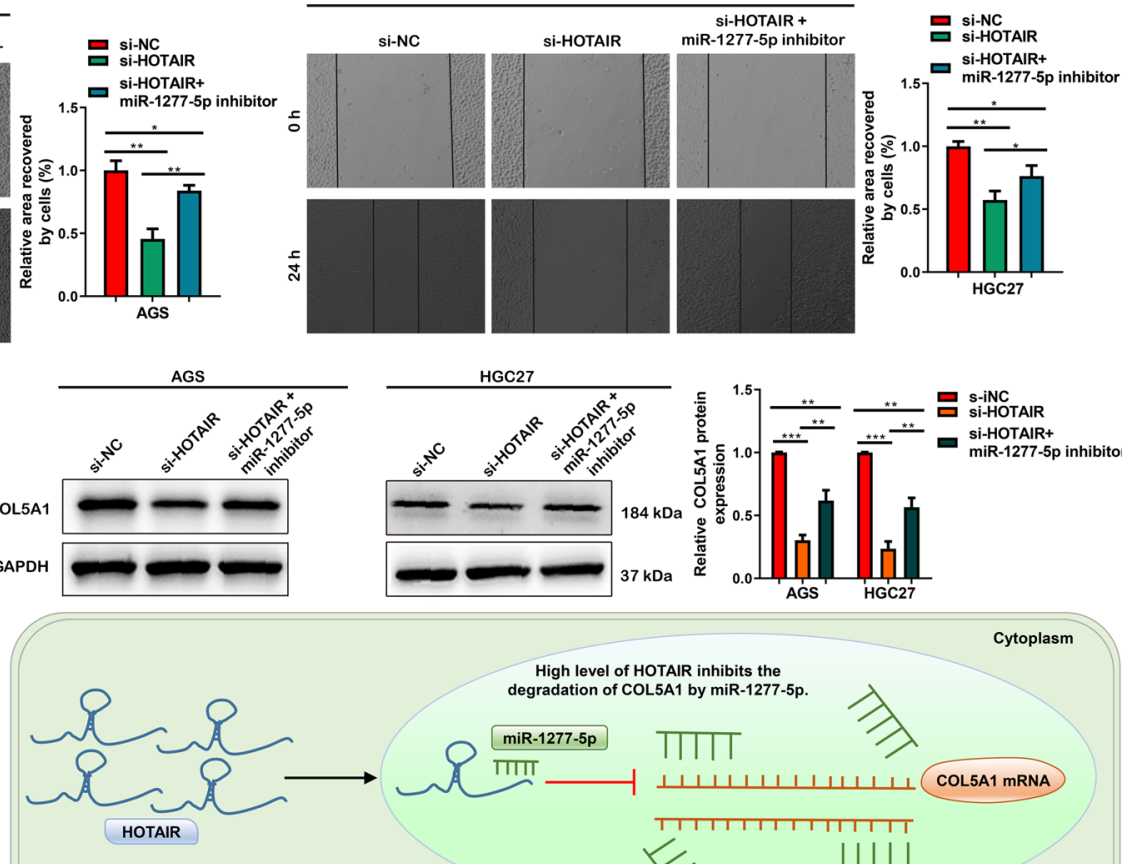

$0.0 \prod_{\mathrm{AGS}} \prod_{\mathrm{HGC27}}$

toplasm
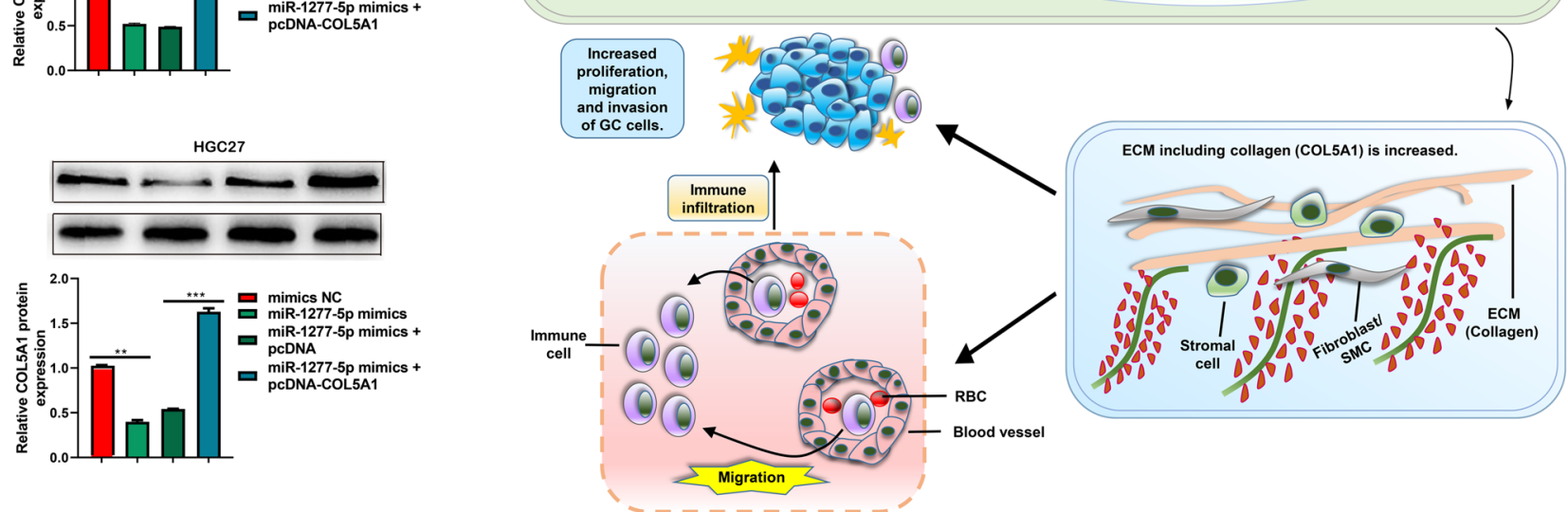

Fig. 6 MiR-1277-5p rescued the oncogenic effects of HOTAIR on GC by regulating COL5A1. a-c Results of EdU and wound healing assays showed that the proliferation and wound healing ability of GC cells was rescued by cotransfection with si-HOTAIR and miR1277-5p inhibitor. d, e Cotransfection with si-HOTAIR and miR1277-5p inhibitor rescued HOTAIR and COL5A1 expression in GC cells. f The pcDNA-COL5A1 restored the effects of miR-1277-5p on COL5A1 expression. $g$ Schematic diagram showing that HOTAIR regulated the proliferation and metastasis of $\mathrm{GC}$ by sponging miR1277-5p by regulating COL5A1. ECM: extracellular matrix; GC: gastric cancer; RBC: red blood cell. Bars represent the mean \pm SD of three independent experiments. ${ }^{*} P<0.05,{ }^{* *} P<0.01,{ }^{* * * *} P<0.001$ 

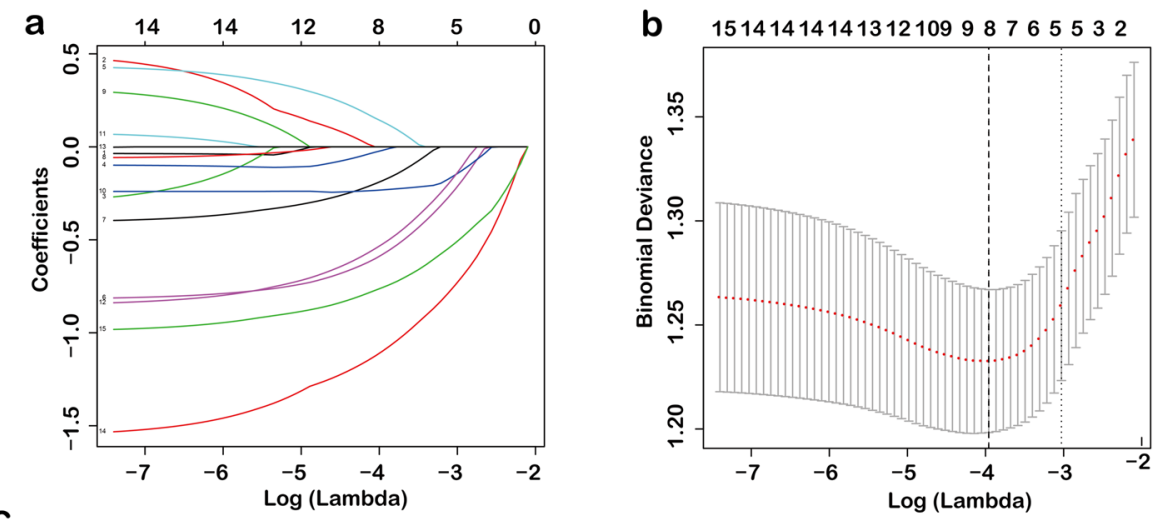

C
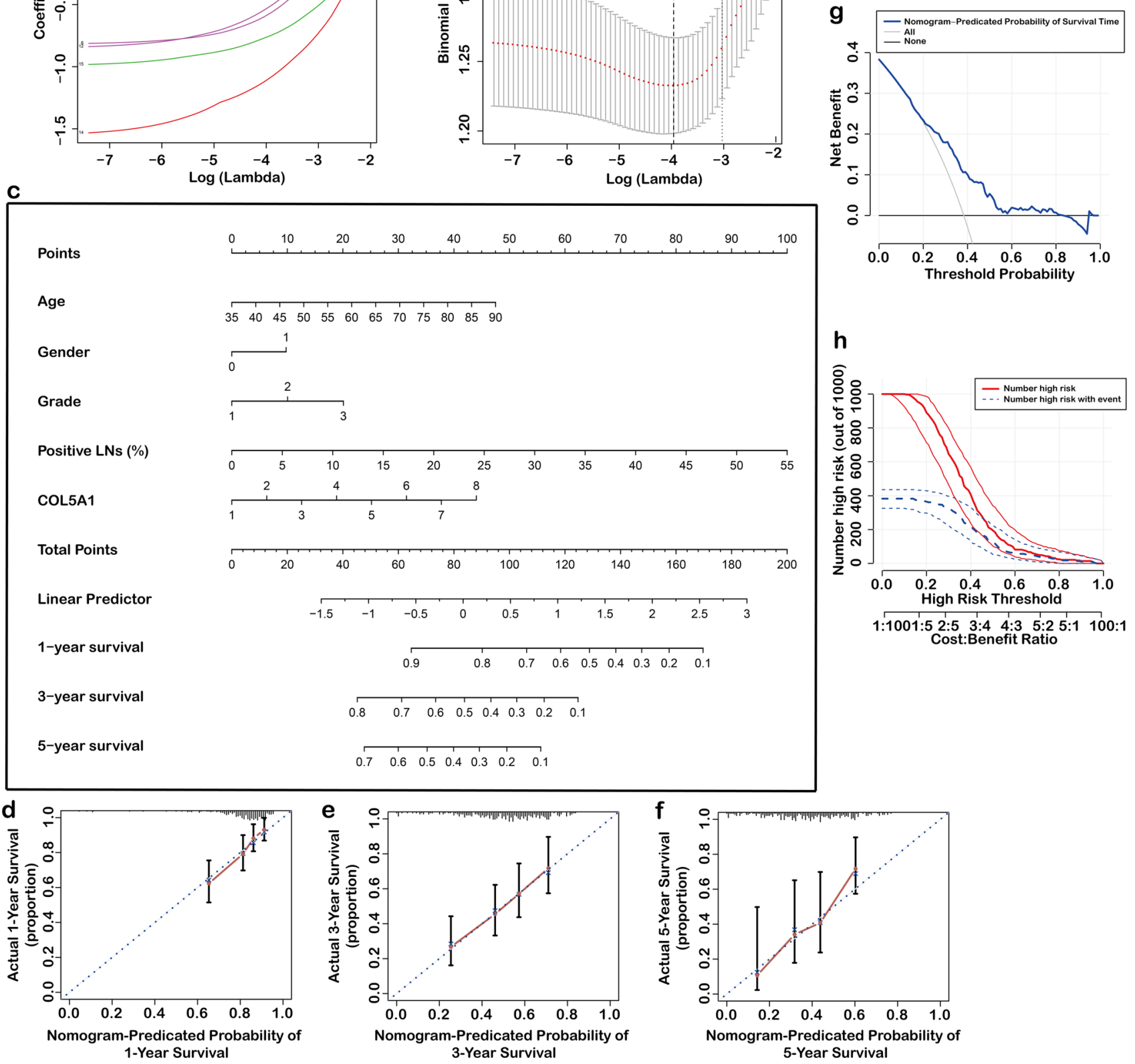

Fig. 7 The COL5A1-based nomogram exerted good performance for predicting the 1/3/5-year survival probability of patients with GC. a, b LASSO regression was used to select 8 clinicopathological characteristics by identifying the optimal lambda value. c A nomogram integrating 5 variables was constructed to predict the $1 / 3 / 5$-year survival

probability of patients with GC. d-f Calibration curves indicated optimal agreement between the predicted 1/3/5-year survival probabilities and the actual survival data. $\mathbf{g}-\mathbf{h}$ Decision curve analysis and clinical impact curve analysis showed that the net benefit was associated with the nomogram-predicted probability

we were able to summarize that increased COL5A1 facilitated immune infiltration, which accelerated the proliferation and metastasis of tumors. Therefore, we speculated that COL5A1-mediated cell proliferation was partially achieved by an increase in immune cell infiltration, which may be regulated by the HOTAIR/miR-1277-5p axis. However, this needs to be further studied for verification. 
To further validate the clinical usefulness of COL5A1, we formulated a COL5A1-based nomogram to predict the OS of GC patients, and this signature incorporated age, gender, tumor grade, positive LNs (\%) and COL5A1 level. Further analyses showed the good agreement, performance, accuracy and net benefit of our model. Hence, COL5A1 may be a prognostic biomarker and new therapeutic target for GC treatment.

In conclusion, we demonstrated that COL5A1 played a vital role in the occurrence and progression of GC, which may be mediated by effects on the tumor microenvironment. In addition, COL5A1-mediated GC cell proliferation and metastasis were regulated by the HOTAIR/miR-1277-5p axis. Our findings imply that ECM could be a potential therapeutic target for GC treatment. However, further study is needed to explore the role of the ECM in tumor growth and immune infiltration, to determine whether the tumor microenvironment is regulated by lncRNA, and to clarify the therapeutic value of these components.

Acknowledgements The results here are partially based upon data generated by the GEO and TCGA Research Network.

Author contributions ZJW and LC performed and analyzed most of the experiments. LM provided intellectual and technical support for the in vitro and in vivo experiments. $\mathrm{ZJW}, \mathrm{LC}, \mathrm{WXH}$, and $\mathrm{AMX}$ oversaw, designed and revised the study and experiments, analyzed the data, and cowrote the manuscript. LH made significant, marked, and substantial contributions to the revision of this work

Funding This work was supported by Natural Science Project of Anhui Province (No. KJ2017A829).

\section{Compliance with ethical standards}

Conflict of interests The authors have declared no potential conflicts of interest.

\section{References}

1. Bray F, Ferlay J, Soerjomataram I, Siegel RL, Torre LA, Jemal A. Global cancer statistics 2018: GLOBOCAN estimates of incidence and mortality worldwide for 36 cancers in 185 countries. CA Cancer J Clin. 2018;68:394-424.

2. Best LMJ, Mughal M, Gurusamy KS. Laparoscopic versus open gastrectomy for gastric cancer. Cochrane DB Syst Rev. 2016:3:CD011389.

3. Wagner AD, Syn NLX, Moehler M, Grothe W, Yong WP, Tai B-C, et al. Chemotherapy for advanced gastric cancer. Cochrane DB Syst Rev. 2017;8:CD004064.

4. Rebekka Schirren DRaARN. Adjuvant and/or neoadjuvant therapy for gastric cancer? A perspective review. Ther Adv Med Oncol. 2015;7:39-48.

5. Gomez-Martín C, Lopez-Rios F, Aparicio J, Barriuso J, GarcíaCarbonero R, Pazo R, et al. A critical review of HER2-positive gastric cancer evaluation and treatment: from trastuzumab, and beyond. Cancer Lett. 2014;351:30-40.
6. Novikova IV, Hennelly SP, Tung C-S, Sanbonmatsu KY. Rise of the RNA machines: exploring the structure of long non-coding RNAs. J Mol Biol. 2013;425:3731-46.

7. Mohammadreza Hajjari AS. HOTAIR: an oncogenic long noncoding RNA in different cancers. Cancer Biol Med. 2015;12:1-9.

8. Liu XHSM, Nie FQ, Ge YB, Zhang EB, Yin DD, Kong R, et al. Lnc RNA HOTAIR functions as a competing endogenous RNA to regulate HER2 expression by sponging miR-331-3p in gastric cancer. Mol Cancer. 2014;13:92.

9. Wang HQR, Guan A, Yao Y, Huang Y, Jia H, Huang W, et al. HOTAIR enhanced paclitaxel and doxorubicin resistance in gastric cancer cells partly through inhibiting miR-217 expression. J Cell Biochem. 2018;119:7226-344.

10. Lu P, Weaver VM, Werb Z. The extracellular matrix: A dynamic niche in cancer progression. J Cell Biol. 2012;196:395-406.

11. Kauppila SSF, Risteli J, Jukkola A, Risteli L. Aberrant type I and type III collagen gene expression in human breast cancer in vivo. J Pathol. 1998;186:262-8.

12. Wozniak MA, Desai R, Solski PA, Der CJ, Keely PJ. ROCKgenerated contractility regulates breast epithelial cell differentiation in response to the physical properties of a three-dimensional collagen matrix. J Cell Biol. 2003;163:583-95.

13. Kaplan G. In vitro differentiation of human monocytes. Monocytes cultured on glass are cytotoxic to tumor cells but monocytes cultured on collagen are not. J Exp Med. 1983;157:2061-72.

14. Liu W, Wei H, Gao Z, Chen G, Liu Y, Gao X, et al. COL5A1 may contribute the metastasis of lung adenocarcinoma. Gene. 2018:665:57-66.

15. Zhang JJ, Yano H, Sasaki T, Matsuo N, Yoshioka H. The proalpha1(V) collagen gene (Col5a1) is coordinately regulated by miR-29b with core promoter in cultured cells. Connect Tissue Res. 2018;59:263-73.

16. Zhao X, Cai H, Wang X, Ma L. Discovery of signature genes in gastric cancer associated with prognosis. Neoplasma. 2016;63:239-45.

17. Li JH, Liu S, Zhou H, Qu LH, Yang JH. starBase v2.0: decoding miRNA-ceRNA, miRNA-ncRNA and protein-RNA interaction networks from large-scale CLIP-Seq data. Nucleic Acids Res. 2014;42:D92-97.

18. Tang Z, Li C, Kang B, Gao G, Li C, Zhang Z. GEPIA: a web server for cancer and normal gene expression profiling and interactive analyses. Nucleic Acids Res. 2017;45:W98-W102.

19. LA Szász AM, Nagy Á, Förster S, Hark K, Green JE, Boussioutas A, et al. Cross-validation of survival associated biomarkers in gastric cancer using transcriptomic data of 1065 patients. Oncotarget. 2016;7:49322-33.

20. Szklarczyk D, Gable AL, Lyon D, Junge A, Wyder S, HuertaCepas J, et al. STRING v11: protein-protein association networks with increased coverage, supporting functional discovery in genome-wide experimental datasets. Nucleic Acids Res. 2019;47:D607-D613613.

21. Yoshihara KSM, Martínez E, Vegesna R, Kim H, Torres-Garcia $\mathrm{W}$, Treviño $\mathrm{V}$, et al. Inferring tumour purity and stromal and immune cell admixture from expression data. Nat Commun. 2013;4:2612.

22. Li T, Fan J, Wang B, Traugh N, Chen Q, Liu JS, et al. TIMER: A Web Server for Comprehensive Analysis of Tumor-Infiltrating Immune Cells. Cancer Res. 2017;77:e108-e110110.

23. Alatengbaolide LD, Li Y, Xu H, Chen J, Wang B, et al. Lymph node ratio is an independent prognostic factor in gastric cancer after curative resection (R0) regardless of the examined number of Lymph Nodes. Am J Clin Oncol. 2013;36:325-30.

24. Bilici A, Selcukbiricik F, Seker M, Oven BB, Olmez OF, Yildiz $\mathrm{O}$, et al. Prognostic significance of metastatic lymph node ratio in patients with pn3 gastric cancer who underwent curative gastrectomy. Oncol Res Treat. 2019;42:209-16. 
25. Lin CL, Zhu GW, Huang YJ, Zheng W, Yang SG, Ye JX. Operable gastric adenocarcinoma with different histological subtypes: Cancer-specific survival in the United States. Saudi J Gastroenterol. 2020;26:46-52.

26. $\mathrm{Li} \mathrm{Z}, \mathrm{Cen} \mathrm{H}$. Construction of a nomogram for the prediction of prognosis in patients with resectable gastric cancer undergoing fewer than sixteen lymph node biopsies. Onco Targets Ther. 2019;12:7415-28.

27. Komatsu S, Ichikawa D, Nishimura M, Kosuga T, Okamoto K, Konishi $\mathrm{H}$, et al. Evaluation of prognostic value and stage migration effect using positive lymph node ratio in gastric cancer. Eur J Surg Oncol. 2017;43:203-9.

28. Kim Y, Squires MH, Poultsides GA, Fields RC, Weber SM, Votanopoulos KI, et al. Impact of lymph node ratio in selecting patients with resected gastric cancer for adjuvant therapy. Surgery. 2017;162:285-94.

29. Yamashita K, Hosoda K, Ema A, Watanabe M. Lymph node ratio as a novel and simple prognostic factor in advanced gastric cancer. Eur J Surg Oncol. 2016;42:1253-60.

30. Guo D, Li Y, Chen Y, Zhang D, Wang X, Lu G, et al. DANCR promotes HCC progression and regulates EMT by sponging miR27a-3p via ROCK1/LIMK1/COFILIN1 pathway. Cell Prolif. 2019;52:e12628.

31. Zhou X, Chen J, Tang W. The molecular mechanism of HOTAIR in tumorigenesis, metastasis, and drug resistance. Acta Bioch Bioph Sin. 2014;46:1011-5.

32. Wanmin Song KJ, McGuire PG. Degradation of type IV collagen by matrix metalloproteinases is an important step in the epithelialmesenchymal transformation of the endocardial cushions. Dev Biol. 2000;227:606-17.

33. Radisky ES, Radisky DC. Matrix metalloproteinase-induced epithelial-mesenchymal transition in breast cancer. J Mammary Gland Biol. 2010;15:201-12.

34. Armstrong TPG, Murphy LB, Bateman AC, Conti JA, Fine DR, Johnson CD, et al. Type I collagen promotes the malignant phenotype of pancreatic ductal adenocarcinoma. Clin Cancer Res. 2004;10:7427-37.
35. Sok JC, Lee JA, Dasari S, Joyce S, Contrucci SC, Egloff AM, et al. Collagen type XI alpha1 facilitates head and neck squamous cell cancer growth and invasion. Br J Cancer. 2013;109:3049-56.

36. Vazquez-Villa F, Garcia-Ocana M, Galvan JA, Garcia-Martinez J, Garcia-Pravia C, Menendez-Rodriguez P, et al. COL11A1/(pro) collagen 11A1 expression is a remarkable biomarker of human invasive carcinoma-associated stromal cells and carcinoma progression. Tumor Biol. 2015;36:2213-22.

37. Feng G, Ma HM, Huang HB, Li YW, Zhang P, Huang JJ, et al. Overexpression of COL5A1 promotes tumor progression and metastasis and correlates with poor survival of patients with clear cell renal cell carcinoma. Cancer Manag Res. 2019;11:1263-74.

38. Ren W, Zhang Y, Zhang L, Lin Q, Zhang J, Xu G. Overexpression of collagen type $\mathrm{V}$ alphal chain in human breast invasive ductal carcinoma is mediated by TGF-beta1. Int $\mathrm{J}$ Oncol. 2018;52:1694-704.

39. Hamy AS, Bonsang-Kitzis H, De Croze D, Laas E, Darrigues L, Topciu L, et al. Interaction between molecular subtypes and stromal immune infiltration before and after treatment in breast cancer patients treated with neoadjuvant chemotherapy. Clin Cancer Res. 2019;25:6731-41.

40. Sokratous G, Polyzoidis S, Ashkan K. Immune infiltration of tumor microenvironment following immunotherapy for glioblastoma multiforme. Hum Vacc Immunother. 2017;13:2575-82.

41. Mao Y, Feng Q, Zheng P, Yang L, Liu T, Xu Y, et al. Low tumor purity is associated with poor prognosis, heavy mutation burden, and intense immune phenotype in colon cancer. Cancer Manag Res. 2018;10:3569-77.

42. Aran DSM, Butte AJ. Systematic pan-cancer analysis of tumour purity. Nat Commun. 2015;6:8971.

Publisher's Note Springer Nature remains neutral with regard to jurisdictional claims in published maps and institutional affiliations. 